\title{
THE MEAN CURVATURE FOR $p$-PLANE
}

\author{
SHUN-ICHI TACHIBANA
}

\section{Introduction}

Let $M$ be an $n$-dimensional Riemannian space. For the skew symmetric tensor $u_{\lambda_{1} \cdots \lambda_{p}}, F_{p}(u)$ for $p=1, \cdots, n$ are defined as follows:

$$
\begin{aligned}
& F_{1}(u)=R_{\lambda \mu} u^{\lambda} u^{\mu}, \\
& F_{p}(u)=R_{\lambda \mu} u^{\lambda \rho_{2} \cdots \rho_{p}} u_{\rho_{2} \cdots \rho_{p}}^{\mu}+\frac{p-1}{2} R_{\lambda \mu \nu \omega} u^{\lambda \mu \rho_{3} \cdots \rho_{p}}{u^{\nu \omega}{ }_{\rho_{3}} \cdots \rho_{p}}_{p}, \quad p \geq 2,
\end{aligned}
$$

where $R_{\lambda \mu \nu \omega}$ is the Riemannian curvature tensor and $R_{\lambda \mu}=R_{\alpha \lambda \mu}{ }^{\alpha}$ is the Ricci tensor of $M$. Throughout this paper indices $\lambda, \mu, \nu, \cdots$ range from 1 to $n$, tensors and vectors will be represented with respect to the natural basis unless stated otherwise, and the summation convention is assumed for these indices. Concerning $F_{p}(u)$ the following theorems are known.

Theorem A [5, p. 64], [3]. If the quadratic form $F_{p}(u)$ is positive definite in a compact Riemannian space, there exists no harmonic p-form other than the zero form.

Theorem B [5, p. 67]. If $F_{p}(u)$ is negative definite in a compact Riemannian space, there exists no Killing tensor field of degree $p$ other than the zero tensor.

Theorem C [4], [2]. If $F_{p}(u)$ is negative definite in a compact Riemannian space for $p \leq n / 2$, there exists no conformal Killing tensor field of degree $p$ other than the zero tensor.

In this paper in $\S 2$ we shall give a geometric meaning of $F_{p}(u)$ in terms of the sectional curvature for a special form $u$ to be called a simple form $u$, and $\S 3$ is devoted to the discussion of the spaces in which $F_{p}(u)$ is independent of the simple form $u$.

\section{Preliminaries}

Let $M$ be an $n$-dimensional Riemannian space. Consider a pair of orthonormal vectors $X=\left(X^{\lambda}\right)$ and $Y=\left(Y^{\lambda}\right)$ at a point $m \in M$. Then the sectional curvature of the 2-plane spanned by $X$ and $Y$ is given by

$$
\rho(X, Y)=-R_{\lambda \mu \nu \omega} X^{\lambda} Y^{\mu} X^{\nu} Y^{\omega} .
$$

Received July 6, 1971, and, in revised form, November 21, 1972. 
Let $\pi$ be a $p$-plane at $m$. An orthonormal base $\left\{e_{i}\right\}, i=1, \cdots, n$, is said to be adapted to $\pi$ if $e_{1}, \cdots, e_{p}$ span $\pi$. Denote $e_{i}=\xi_{i}{ }^{2} \partial / \partial x^{2}$, and define

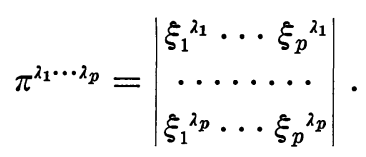

Consider another orthonormal base $\left\{e_{i}^{\prime}\right\}$ adapted to $\pi$. Then

$$
e_{i}^{\prime}=\sum_{j=1}^{p} a_{i j} e_{j}, \quad i=1, \cdots, p,
$$

where the $p \times p$ matrix $A=\left(a_{i j}\right)$ is orthogonal. Thus under the change of adapted bases we have

$$
\pi^{\prime \lambda_{1} \cdots \lambda_{p}}= \pm \pi^{\lambda_{1} \cdots \lambda_{p}}
$$

and $\pi^{\lambda_{1} \cdots \lambda_{p}}$ is determined for $\pi$ within a sign. We shall call the tensor $\pi^{\lambda_{1} \cdots \lambda_{p}}$ the simple $p$-vector of $\pi$, and denote it by $\pi$ again. The ambiguity of signs does not matter in the following discussion.

\section{The mean curvature for $\pi$}

Let $\pi$ be a $p$-plane at $m$, and $\left\{e_{i}\right\}$ an adapted base. Put

$$
\rho\left(\pi_{e}\right)=\frac{1}{p(n-p)} \sum_{i=1}^{p} \sum_{j=p+1}^{n} \rho\left(e_{i}, e_{j}\right)
$$

and prove that its value depends only on $\pi$. In fact, it will be seen as follows that $\rho\left(\pi_{e}\right)$ is independent of the choice of $\left\{e_{i}\right\}$.

Let $F_{p}(u)$ be the quadratic form of $u$ defined in the introduction. Denote by $F_{p}\left(\pi_{e}\right)$ the $F_{p}(u)$ with $u^{\lambda_{1} \cdots \lambda_{p}}$ to be $\pi^{\lambda_{1} \cdots \lambda_{p}}$ of (1.1), and define

$$
\bar{\rho}\left(\pi_{e}\right)=\frac{1}{p !(n-p)} F_{p}\left(\pi_{e}\right)
$$

which is independent of the choice of adapted bases to $\pi$, because of (1.2). Thus for our purpose mentioned above it is sufficient to show that $\rho\left(\pi_{e}\right)=$ $\bar{\rho}\left(\pi_{e}\right)$.

As $F_{p}\left(\pi_{e}\right)$ is a tensor equation, we may consider it written with respect to the adapted base $\left\{e_{i}\right\}$ of $\pi$. Then the components of $e_{i}$ are $\delta_{i}{ }^{2}$, and the simple $p$-vector has the components

$$
\pi^{\lambda_{1} \cdots \lambda_{p}}= \begin{cases}\operatorname{sign}\left(\lambda_{1}, \cdots, \lambda_{p}\right), & \text { if }\left(\lambda_{1}, \cdots, \lambda_{p}\right) \text { is a permutation of }\{1, \cdots, p\}, \\ 0 & \text { other cases. }\end{cases}
$$


Thus we have for $\lambda, \mu, \nu, \omega=1, \cdots, p$

$$
\begin{aligned}
\pi^{\lambda \rho_{2} \cdots \rho_{p}} \pi_{\rho_{2} \cdots \rho_{p}}^{\mu} & =(p-1) ! \delta_{\lambda \mu}, \\
\pi^{\lambda \mu \rho_{3} \cdots \rho_{p}} \pi_{\rho_{3} \cdots \rho_{p}} & =(p-2) !\left(\delta_{\lambda \nu} \delta_{\mu \omega}-\delta_{\lambda \omega} \delta_{\mu \nu}\right),
\end{aligned}
$$

and the following equations are valid:

$$
\begin{aligned}
R_{\lambda \mu} \pi^{\lambda \rho_{2} \cdots \rho_{p}} \pi_{\rho_{2} \cdots \rho_{p}}^{\mu}=(p-1) ! & \sum_{\lambda=1}^{p} R_{\lambda \lambda}=(p-1) ! \sum_{\lambda=1}^{p} \sum_{\mu=1}^{n} \rho\left(e_{\lambda}, e_{\mu}\right) \\
\frac{p-1}{2} R_{\lambda \mu \nu \omega} \pi^{\lambda \mu \rho_{3} \cdots \rho_{p}} \pi_{\rho_{3} \cdots \rho_{p}}^{\nu \omega} & =(p-1) ! \sum_{\lambda, \mu=1}^{p} R_{\lambda \mu \lambda \mu} \\
& =-(p-1) ! \sum_{\lambda=1}^{p} \sum_{\mu=1}^{p} \rho\left(e_{\lambda}, e_{\mu}\right) .
\end{aligned}
$$

Hence $\bar{\rho}\left(\pi_{e}\right)=\rho\left(\pi_{e}\right)$. Since $\rho\left(\pi_{e}\right)$ depends only on $\pi$, we denote it by $\rho(\pi)$ and call it the mean curvature for the $p$-plane $\pi$. We notice that the mean curvature for the $p$-plane spanned by $e_{1}, \cdots, e_{p}$ coincides with that for the $(n-p)$-plane spanned by $e_{p+1}, \cdots, e_{n}$.

\section{A theorem analogous to Schur's theorem}

In this section we shall determine the spaces in which $\rho(\pi)$ is independent of the $p$-plane $\pi$ at each point. First we have

Lemma 1. Let $A=\left(a_{i j}\right)$ be an $n \times n$ symmetric matrix whose diagonal elements are all zero. If $A$ satisfies

$$
\sum_{h, k=1}^{p} a_{i_{h}, i_{k}}=0
$$

for any $i_{1}<\ldots<i_{p}$ taken from $\{1, \cdots, n\}$ and $n-1>p>1$, then $A$ is the zero matrix.

Proof. For $\left\{i_{1}, \cdots, i_{p}\right\}=\{1, \cdots, p\}$ and $\left\{i_{1}, \cdots, i_{p}\right\}=\{2, \cdots, p+1\}$ from (3.1) we have, respectively,

$$
\sum_{i, j=1}^{p} a_{i j}=0, \quad \sum_{i, j=2}^{p+1} a_{i j}=0
$$

which imply, due to $a_{i j}=a_{j i}$, that

$$
\sum_{i=1}^{p+1} a_{i 1}=\sum_{i=1}^{p+1} a_{i, p+1}
$$

Similarly,

$$
\sum a_{i 1}=\sum a_{i 2}=\cdots=\sum a_{i p}=\sum a_{i, p+1},
$$


where $\sum$ denotes the summation over $i$ from 1 to $p+1$. If we use $p+2$ instead of $p+1$, then

$$
\Sigma^{\prime} a_{i 1}=\Sigma^{\prime} a_{i 2}=\cdots=\Sigma^{\prime} a_{i p}=\Sigma^{\prime} a_{i, p+2},
$$

where $\Sigma^{\prime}$ denotes the summation over $i$ from 1 to $p$ and $p+2$. Therefore we get

$$
\begin{aligned}
a_{p+1,1}-a_{p+2,1} & =a_{p+1,2}-a_{p+2,2}=\cdots=a_{p+1, p}-a_{p+2, p} \\
& =\sum a_{i, p+1}-\sum^{\prime} a_{i, p+2}=\sum a_{p+1, i}-\sum^{\prime} a_{p+2, i} .
\end{aligned}
$$

If we denote the above common value by $k$, then

$$
p k=\sum_{i=1}^{p}\left(a_{p_{+1, i}}-a_{p_{+2, i}}\right)=\sum a_{p_{+1, i}}-\sum^{\prime} a_{p_{+2, i}}=k,
$$

from which follows $k=0$. Thus we have

$$
a_{p+1, i}=a_{p+2, i}, \quad i=1, \cdots, p .
$$

Similarly,

$$
a_{p+1, i}=a_{p_{+2, i}}=\cdots=a_{n i}, \quad i=1, \cdots, p .
$$

The similar process for $\{1, \cdots, p-1, p+1\}$ leads us to

$$
a_{p i}=a_{p+2, i}=\cdots=a_{n i}, \quad i=1, \cdots, p-1, p+1,
$$

and finally we get

$$
a_{i j}=k_{j}, \quad j=1, \cdots, p ; \quad i=1, \cdots, n ; \quad i \neq j .
$$

As $a_{i j}=a_{j i}$, we obtain $a_{i j}=c$ for $i, j=1, \cdots, p$, and hence $c=0$ follows from (3.2). In a similar way, we know all the elements of $A$ to be zero. q.e.d.

Now let us assume that $\rho(\pi)$ is independent of the $p$-plane at each point and takes the value $k /(n-p)$, where $k$ is a scalar function. By the assumption we have $F_{p}\left(\pi_{e}\right)=p ! k$, and hence

$$
L_{\lambda \mu \nu \omega} \pi^{\lambda \mu \rho_{3} \cdots \rho_{p}} \pi_{\rho_{3} \cdots \rho_{p}}=0
$$

on- taking account of (2.1), where

$$
\begin{aligned}
L_{\lambda \mu \nu \omega}= & (p-1) R_{\lambda \mu \nu \omega}-k\left(g_{\lambda \nu} g_{\mu \omega}-g_{\lambda \omega} g_{\mu \nu}\right) \\
& +\frac{1}{2}\left(R_{\lambda \nu} g_{\mu \omega}-R_{\lambda \omega} g_{\mu \nu}+R_{\mu \omega} g_{\lambda \nu}-R_{\mu \nu} g_{\lambda \omega}\right) .
\end{aligned}
$$

Now we may consider that (3.3) has been written with respect to the adapted base of (1.1). Then by virtue of (2.1) we get 


$$
\sum_{\lambda, \mu=1}^{p} L_{\lambda \mu \lambda \mu}=0
$$

for the base. Similarly, the analogous equations are valid for any $p$ indices.

Thus, if we put $a_{\lambda \mu}=L_{\lambda \mu \lambda \mu},(\lambda, \mu=1, \cdots, n)$, then the $n \times n$ matrix $A=\left(a_{\lambda \mu}\right)$ satisfies the condition of Lemma 1 ; consequently $a_{\lambda \mu}=0$ follows.

On the other hand, we know [1, p. 196]

Lemma 2. Let $L$ be a tensor of type $(0,4)$ satisfying

$$
L_{\lambda \mu \nu \omega}=-L_{\mu \lambda \nu \omega}=-L_{\lambda \mu \omega \nu}, \quad L_{\lambda \mu \nu \omega}+L_{\mu \nu \lambda \omega}+L_{\nu \lambda \mu \omega}=0 .
$$

If $L_{\lambda \mu \lambda \mu}$ for all $\lambda$ and $\mu$ with respect to any orthonormal base are zero, then $L$ is the zero tensor.

The tensor $L_{\lambda \mu \nu \omega}$ of (3.4) clearly satisfies the condition of Lemma 2. Thus we get

$$
L_{\lambda \mu \nu \omega}=0 \text {. }
$$

Transvecting $g^{2 \omega}$ with the last equation, we have

$$
(2 p-n) R_{\mu \nu}=[R-2 k(n-1)] g_{\mu \nu},
$$

where $R=g^{\lambda \omega} R_{\lambda \omega}$ is the scalar curvature. If $2 p \neq n$, it follows that

$$
k=\frac{n-p}{n(n-1)} R, \quad R_{\mu \nu}=\frac{R}{n} g_{\mu \nu},
$$

and substituting these values into (3.5) we get

$$
R_{\lambda \mu \nu \omega}=\frac{R}{n(n-1)}\left(g_{\lambda \omega} g_{\mu \nu}-g_{\lambda \nu} g_{\mu \omega}\right),
$$

which shows $M$ to be a space of constant curvature, provided that $n>2$.

When $n=2 p$, from (3.6) it follows that

$$
k=\frac{R}{2(n-1)},
$$

and (3.5) becomes

$$
\begin{gathered}
(n-2) R_{\lambda \mu \nu \omega}+R_{\lambda \nu} g_{\mu \omega}-R_{\lambda \omega} g_{\mu \nu}+R_{\mu \omega} g_{\lambda \nu}-R_{\mu \nu} g_{\lambda \omega} \\
-\frac{R}{n-1}\left(g_{\lambda \nu} g_{\mu \omega}-g_{\lambda \omega} g_{\mu \nu}\right)=0
\end{gathered}
$$

which shows $M$ to be conformally flat, provided that $n>3$.

Thus we have the following theorem including the trivial cases where $p=1$ 
and $n-1$; the converse part is proved by making use of $F_{p}\left(\pi_{e}\right)$.

Theorem. In an $n$-dimensional Riemannian space $M$, if the mean curvature for p-plane is independent of the p-plane at each point, then

(i) $M$ is an Einstein space, for $p=1, n-1$ and $n>2$,

(ii) $M$ is of constant curvature, for $n-1>p>1$ and $2 p \neq n$,

(iii) $M$ is conformally flat, for $n-1>p>1$ and $2 p=n$.

The converse is also true.

\section{Bibliography}

[1] E. Cartan, Leçon sur les géométrie des espaces de Riemann, 2nd ed., GauthierVillars, Paris, 1951.

[2] T. Kashiwada, On conformal Killing tensor, Natur. Sci. Rep. Ochanomizu Univ. 19 (1968) 67-74.

[ 3 ] I. Mogi, On harmonic fields in Riemannian manifold, Kōdai Math. Sem. Rep. 2 (1950) 61-66.

[4] S. Tachibana, On conformal Killing tensor in a Riemannian space, Tôhoku Math. J. 21 (1969) 56-64.

[5] K. Yano \& S. Bochner, Curvature and Betti numbers, Annal of Math. Studies, No. 32, Princeton University Press, Princeton, 1953.

OChANOMIZU University, TOKYo 